\title{
A Multicenter, Randomized, Double-Blind, Placebo-Controlled Trial Assessing Efficacy and Safety of a Novel Low-Dose Turmeric Extract Formulation in Healthy Adults with Chronic Knee Pain
}

\author{
Shefali Thanawala (D) \\ Rajat Shah iD ' \\ Venkateswarlu Somepalli (D) ${ }^{2}$ \\ KrishnaRaju Venkata Alluri (iD ${ }^{2}$ \\ Prabakaran Desomayanandam (iD ${ }^{3}$ \\ Arun Bhuvanendran (iD ${ }^{3}$ \\ 'Inventia Healthcare Ltd., Mumbai, \\ Maharashtra, India; ' 2 Laila Nutraceuticals, \\ Vijayawada, Andhra Pradesh, India; ${ }^{3}$ In \\ vitro Research Solutions (iVRS) Pvt Ltd, \\ Bengaluru, Karnataka, India
}

Background: Knee pain causes functional limitations, eventually compromising the quality of life. We evaluated the efficacy of our water-dispersible turmeric formulation $(60 \%$ natural curcuminoids, TurmXTRA $60 \mathrm{~N}^{\mathbb{R}}$-WDTE60N), which exhibited better PK profile at low dose $(250 \mathrm{mg})$ than standard turmeric extract, in alleviating symptoms of chronic knee pain. Methods: In this randomized, double-blind, placebo-controlled trial, subjects received either $250 \mathrm{mg}$ WDTE60N capsule (150 mg curcuminoids; $\mathrm{n}=53$ ) or appearance-matched placebo capsule $(n=53)$ once daily for 90 days. Primary endpoint was change in pain score on the visual analogue scale (VAS) after 80 -m fast-paced walk test.

Results: A total of 96 subjects completed the study. WDTE60N reduced VAS score from baseline $(5.4 \pm 0.9)$ to day $90(3.8 \pm 0.8)$ with greater mean reduction than placebo $(-1.5 \pm$ 0.7 vs $-0.6 \pm 0.8, p<0.0001 ; 2.5$ times). It also significantly improved the time taken for 80 $\mathrm{m}$ fast-paced walk test and 9-step stair-climb test; and improved all biomarkers compared to placebo $(p>0.05)$. Three adverse events occurred but were unrelated to study products.

Conclusion: WDTE60N $250 \mathrm{mg}$ administered once daily for 3 months, alleviated knee pain, improved joint function in healthy subjects with chronic knee pain, was well tolerated and safe.

Keywords: biomarkers, chronic pain, curcuminoids, knee joint, turmeric, visual analog scale

\section{Introduction}

Chronic knee pain is one of the most common reasons for medical consultation at primary care centers. People with knee pain experience functional limitations that compromise their quality of life substantially. ${ }^{1,2}$ Annual prevalence of knee pain in adults $>50$ years old was estimated to be approximately $50 \% .{ }^{3}$ Osteoarthritis (OA) is a common cause of chronic knee pain ${ }^{4}$ and is characterized by progressive loss of structure and function of the articular cartilage. Early life factors such as anterior knee pain syndrome in adolescence possibly lead to the development of knee OA in late $40 \mathrm{~s}^{5}$ Inflammation plays a critical role in the pathogenesis of OA. ${ }^{6}$ In early OA, increase in the expression of inflammatory mediators in the synovium amplifies catabolic activity, consequently promoting cartilage degradation. ${ }^{7}$ In this prodromal stage, although subjects remain asymptomatic with optimal functional status, the underlying cellular processes are actively damaging the joint. ${ }^{8}$ Anti-inflammatory
Correspondence: Shefali Thanawala Inventia Healthcare Ltd., Mumbai, Maharashtra, India

Tel +9l 9324210054

Email shefali.

thanawala@inventiahealthcare.com 


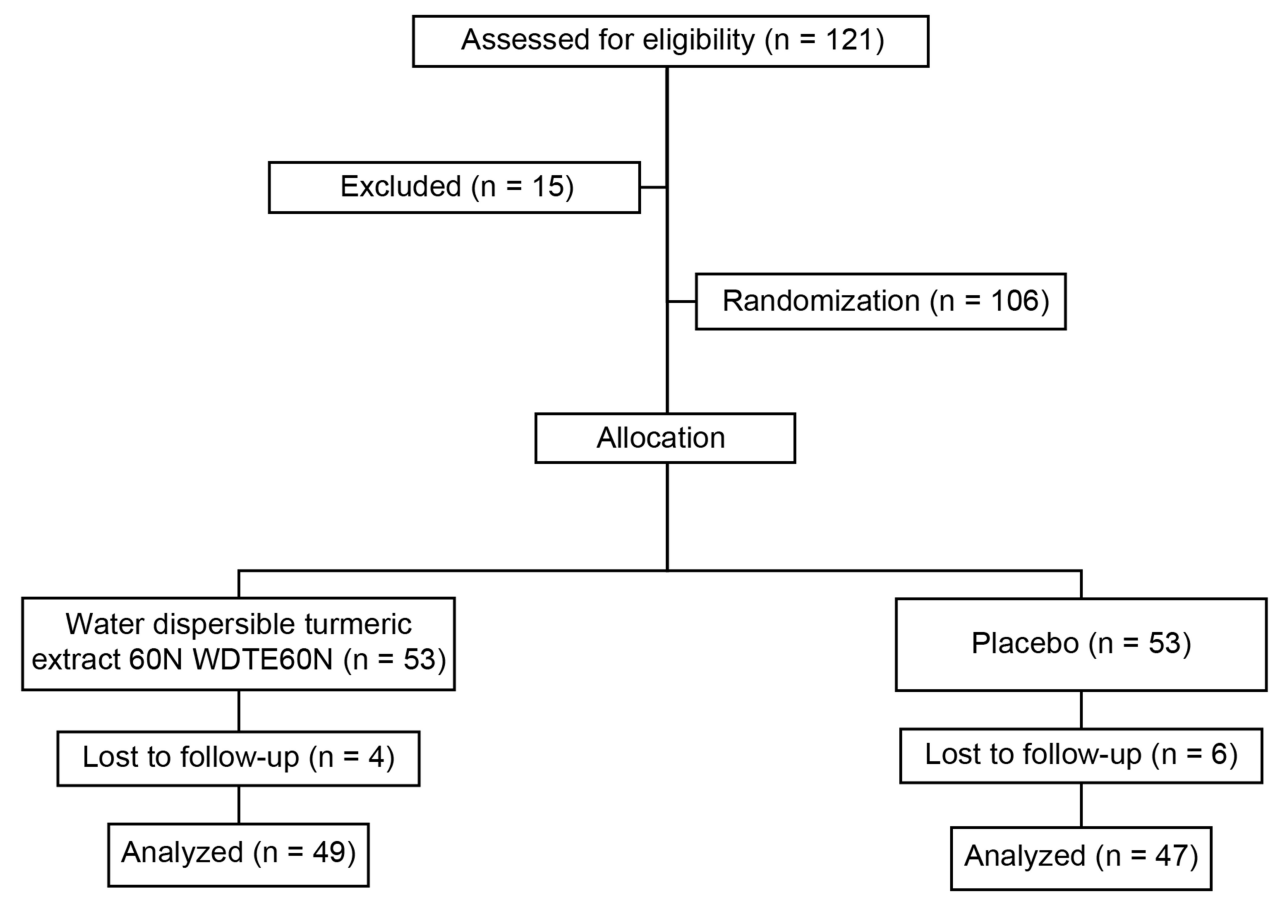

Figure I Subject flow diagram.

drugs administered at this early stage of OA reportedly have the potential to prevent disease progression. ${ }^{9}$ Non-steroidal anti-inflammatory drugs (NSAIDs) remain the first-line of treatment for knee pain associated with OA. ${ }^{10}$ However, these drugs are known to exert a range of cardiovascular, renal, gastrointestinal, and hematological adverse events (AEs). ${ }^{11,12}$ Moreover, age-related decline in the kidney and liver functions intensifies the risk of AEs and drugdrug interactions. ${ }^{13}$ Studies have reported dissatisfaction among NSAID users with respect to adequate pain relief. ${ }^{14,15}$ Also, NSAIDs, while causing the known adverse effects, do not halt disease progression. ${ }^{16}$ In this scenario, supplementation of nutrients, natural or synthetic, is a recent strategy for managing and preventing OA. ${ }^{17}$ Various nutraceutical ingredients have been evaluated for treating $\mathrm{OA}$

Table I Baseline Demographic Characteristics

\begin{tabular}{|l|l|l|}
\hline & $\begin{array}{l}\text { WDTE60N } \\
(\mathbf{n}=\mathbf{5 3})\end{array}$ & $\begin{array}{l}\text { Placebo } \\
(\mathbf{n}=\mathbf{5 3})\end{array}$ \\
\hline Age (year) & $36.6 \pm 10$ & $37.7 \pm 9.3$ \\
\hline Gender & & \\
Men & 24 & 30 \\
Women & 29 & 23 \\
\hline Body mass index $\left(\mathrm{kg} / \mathrm{m}^{2}\right)$ & $23.7 \pm 2.8$ & $23.6 \pm 2.4$ \\
\hline
\end{tabular}

pain. ${ }^{18,19}$ A few natural botanical nutritional interventions have shown their potential to regulate the balance between anabolic and catabolic processes in joint tissue via their free-radical scavenging and potent anti-inflammatory actions. $^{20}$ Turmeric extract and curcumin are one of the botanical supplements used frequently for joint conditions. Curcumin has been found to be one of the most effective agents for pain reduction in patients with $\mathrm{OA}$ in two recent meta-analyses that evaluated a large number of dietary supplements. ${ }^{21,22}$

Curcuminoids are the principal components of the culinary spice plant, turmeric. Scientifically known as Curcuma longa L., turmeric is used as a condiment and flavoring agent in the food industry. ${ }^{23}$ Turmeric has been used since ancient times as an anti-inflammatory agent in traditional Chinese, and Ayurvedic systems of medicines. The plant was known in Traditional Persian Medicine as a useful remedy for different inflammations and pain. When used as alternative medicine or as a dietary supplement, turmeric is typically used as an extract standardized to $80-95 \%$ curcuminoids, primarily containing curcumin. $^{24,25}$ The curcuminoids comprise three actives, viz., curcumin, bisdemethoxycurcumin (BDMC), and demethoxycurcumin (DMC) that exist as soluble ketoenol tautomers. ${ }^{26,27}$ Curcuminoids exert a broad spectrum 
Table 2 Efficacy Endpoints

\begin{tabular}{|c|c|c|c|c|c|}
\hline & \multicolumn{2}{|c|}{ WDTE60N (n = 49) } & \multicolumn{2}{|c|}{ Placebo $(n=47)$} & \multirow[t]{2}{*}{$p$-value } \\
\hline & Mean \pm SD & Median (IQR) & Mean \pm SD & Median (IQR) & \\
\hline \multicolumn{6}{|c|}{ Visual analogue scale after $80-\mathrm{m}$ fast-paced walk test } \\
\hline Baseline & $5.4 \pm 0.9$ & $5(5,6)$ & $5.4 \pm 0.8$ & $5(5,6)$ & \\
\hline Day 45 & $4.4 \pm 0.8$ & $4(4,5)$ & $4.9 \pm 0.6$ & $5(5,5)$ & \\
\hline Day 90 & $3.8 \pm 0.8$ & $4(3,4)$ & $4.8 \pm 0.6$ & $5(4.3,5)$ & \\
\hline Change from $B$ to $D 45$ & $-1.5 \pm 0.4$ & $-I(-I,-I)$ & $-0.4 \pm 0.6$ & $0(-1,0)$ & $<0.0001 *$ \\
\hline Change from $B$ to $D 90$ & $-1.5 \pm 0.7$ & $-2(-2,-1)$ & $-0.6 \pm 0.8$ & $0(-1,0)$ & $<0.000 I^{*}$ \\
\hline \multicolumn{6}{|c|}{$80 \mathrm{~m}$ - fast-paced walk test (s) } \\
\hline Baseline & $70.3 \pm 23.9$ & $60.1(59.1,60.5)$ & $69.2 \pm 23.4$ & $60.08(57.7,60.3)$ & \\
\hline Day 45 & $65.2 \pm 18.8$ & $60.0(58.9,60.1)$ & $62.9 \pm 15.2$ & $60.04(57.8,60.2)$ & \\
\hline Day 90 & $59.3 \pm 9.5$ & $59.8(57.3,60.1)$ & $62.4 \pm 15.4$ & $60.0(57.4,60.1)$ & \\
\hline Change from B to D45 & $-5.0 \pm 16.5$ & $-0.09(-0.3,-0.04)$ & $-6.3 \pm 18.6$ & $0.0(-0.07,0.07)$ & $0.0002 *$ \\
\hline Change from $B$ to $D 90$ & $-11.0 \pm 26.1$ & $-0.2(-2,-0.06)$ & $-6.8 \pm 23.3$ & $0(-0.2,0.2)$ & $0.001 I^{*}$ \\
\hline \multicolumn{6}{|l|}{ 9-step stair climb test (s) } \\
\hline Baseline & $49.2 \pm 40.9$ & $60.2(10.8,60.5)$ & $44.8 \pm 39.2$ & $60.2(10.6,60.5)$ & \\
\hline Day 45 & $40.2 \pm 29.7$ & $60.0(10.6,60.2)$ & $39.9 \pm 32.1$ & $60.1(10.8,60.2)$ & \\
\hline Day 90 & $36.9 \pm 24.3$ & $52.0(10.5,60.1)$ & $37.0 \pm 27.6$ & $52(10.8,60.1)$ & \\
\hline Change from $B$ to D45 & $-8.9 \pm 21.0$ & $-0.1(-0.4,-0.07)$ & $-4.9 \pm 21.0$ & $0.04(-0.07,0.09)$ & $<0.000 I^{*}$ \\
\hline Change from $B$ to $D 90$ & $-12.3 \pm 24.2$ & $0.2(-0.8,-0.1)$ & $-7.8 \pm 24.2$ & $0.04(-0.2,0.1)$ & $<0.0001 *$ \\
\hline \multicolumn{6}{|c|}{ Primary knee flexion (degree) } \\
\hline Baseline & $134.2 \pm 0.9$ & $134(134,135)$ & $134.4 \pm 0.9$ & $134(134,135)$ & \\
\hline Day 45 & $134.1 \pm 0.6$ & $134(134,134)$ & $134.3 \pm 0.8$ & $134(134,135)$ & \\
\hline Day 90 & $134.0 \pm 0.6$ & $134(134,134)$ & $134.3 \pm 0.7$ & $134(134,134)$ & \\
\hline Change from B to D45 & $-0.1 \pm 0.7$ & $0(0,0)$ & $-0.0 \pm 0.5$ & $0(0,0)$ & \\
\hline Change from $B$ to $D 90$ & $-0.2 \pm 0.6$ & $0(0,0)$ & $-0.1 \pm 0.8$ & $0(0,0)$ & 0.3657 \\
\hline
\end{tabular}

Note: * $p$-value less than $0.05(p \leq 0.05)$ is statistically significant.

Abbreviations: $B$ to $D$, baseline to day; IQR, interquartile range.

of biological activities including substantial antiinflammatory activity. In vitro evidence shows that curcuminoids exert anti-apoptotic effects on interleukin (IL)-1 $\beta$ stimulated human articular chondrocytes, ${ }^{28}$ inhibit proinflammatory mediators, including prostaglandin E2, ${ }^{29}$ nitric oxide, IL-6, and IL-8; and inhibit the production of matrix metalloproteinase- 1 and $3 .^{30,31}$

Despite the proven benefits of curcumin, its poor bioavailability has been a limiting factor. When administered orally, only a small amount of curcumin is absorbed, thus requiring multiple daily doses for gaining optimal effects. $^{32}$ Such a high dosage is associated with poor compliance, particularly when used long term. Moreover, studies also showed that the incidence of AEs like gastrointestinal disturbances and urticaria increases with increase in dosage. ${ }^{33}$ Thus, to overcome these limitations, that is, to provide optimum therapeutic effects of curcuminoids at a lower dose, a novel formulation of the natural, waterdispersible turmeric extract containing $60 \%$ natural curcuminoids (TurmXTRA $60 \mathrm{~N}^{\circledR}$ - WDTE60N; of Inventia Healthcare Ltd. and Laila Nutraceuticals, India) was developed using a patented technology. This formulation has been thoroughly evaluated in pre-clinical and human pharmacokinetics (PK) studies. In the preclinical PK study, when $300 \mathrm{mg} / \mathrm{kg}$ of curcuminoids were administered to Sprague Dawley rats, the bioavailability of WDTE60N was found to be 10 times higher than the standard turmeric extract $95 \% .{ }^{34}$ Applying these results, further based on translational research, a comparative PK study in healthy adult volunteers was conducted to evaluate the bioavailability of WDTE60N at a 10 times lower dose (250 mg, containing $150 \mathrm{mg}$ of curcuminoids), as that of the frequently used dose of standard turmeric extract 95\% (containing $1500 \mathrm{mg}$ of curcuminoids). The area 
under the blood concentration-time curve were similar and peak plasma concentration was higher for total curcumin after oral administration of WDTE60N at a 10-fold lower dose compared to standard turmeric extract. These findings demonstrate significantly higher bioavailability of WDTE60N compared to the currently available standard curcumin preparations. Thus, WDTE60N is believed to provide clinical benefits of curcumin in humans at a 10 times lower single daily dose of $150 \mathrm{mg}$ of active curcuminoids.

Given the favorable results in our previous PK studies, we conducted a randomized controlled trial to evaluate the efficacy and safety of our low-dose formulation-WDTE60N-in improving joint comfort by alleviating chronic knee pain. We hypothesized that WDTE60N supplementation in a single daily dose of $250 \mathrm{mg}$ for 3 months would improve the symptoms and functional abilities in healthy subjects with chronic knee pain with no major side effects.

\section{Materials and Methods}

\section{Clinical Study Design and Study Population}

A randomized, double-blind, placebo-controlled clinical trial was conducted in healthy adult subjects who experienced chronic knee pain following physical exertion. This study was performed at medical facilities in India (Sri Venkateshwara Hospital, Shetty's Hospital, and Surya Medical Centre, Bangalore) from March 2020 to September 2020. The study protocol was approved on 18 February, 2020 by the Ethics Committee of Sri Venkateshwara Hospital Ethics Committee and Shetty's Hospital Ethics Committee (PRO-INV-012-019). The study was conducted in accordance with the Good Clinical Practice (GCP) and the Declaration of Helsinki, as well as the Schedule Y (amended version 2005) and Indian Council of Medical Research codes. The study protocol was registered with the Clinical Trial Registry of India (CTRI/2020/03/023877). All subjects provided written informed consent prior initiating clinical studyrelated activities.

The study included men and women aged between 18 and 60 years, with a body mass index (BMI) of 18 to $30 \mathrm{~kg} / \mathrm{m}^{2}$ having chronic knee pain for at least 2 months. Subjects were eligible if they had moderate pain, ie, VAS score of 4 or greater in the knee joint and were willing to limit the use of analgesic and/or anti-inflammatory drugs only as a rescue medication until study completion. Exclusion criteria included pregnant, lactating, or postmenopausal women; subjects with knee arthritis detected on radiography; those taking more than two analgesic drugs and/or anti-inflammatory agents daily for more than 3 months; and those with a history of surgery or trauma affecting the knee, impaired renal or liver function, or known history of any chronic illness.

\section{Study Procedure and Study Treatments}

The study involved four visits: visit 1 (screening visit), visit 2 or baseline (randomization visit), visit 3 (first follow-up at day 45), and visit 4 (final follow-up visit at day 90). Block randomization schedule was generated using PROC PLAN in SAS $^{\circledR}$ version 9.4 (SAS Institute Inc., USA) by an independent statistician. The investigator allocated the study treatments to subjects as per the randomization schedule. The study products were stored in containers with appropriate labeling. The statistician blinded the samples by sticking a blinded label according to the randomization procedures against unique codes.

Eligible subjects were randomized to receive either WDTE60N capsules or inert placebo capsules having identical appearance. The WDTE60N capsules contained $150 \mathrm{mg}$ of curcuminoids including curcumin, demethoxycurcumin, and bisdemethoxycurcumin. Study subjects were given a bottle of their respective study product (52 capsules for 45 days) at visits 2 and 3, and were instructed to take one capsule per day orally with water after breakfast.

Paracetamol tablet $650 \mathrm{mg}$ or diclofenac sodium tablet $50 \mathrm{mg}$, along with explanation of the dose and frequency by the treating physician, was provided as rescue medication that was to be used only if the subject's knee pain worsened during the study.

\section{Efficacy Assessments}

The study objective was to evaluate the efficacy of WDTE60N in improving joint function and alleviating joint pain in healthy non-arthritic subjects. The primary endpoint was reduction in the pain intensity level from baseline to day 90 as assessed by the VAS after the $80-\mathrm{m}$ fast-paced walk test. Secondary efficacy measures included performance in the 80 -m fast-paced walk test, 9-step stair climb test (SCT), and range of motion test (primary knee flexion). We also measured changes from baseline to day 90 in following inflammatory markers: 


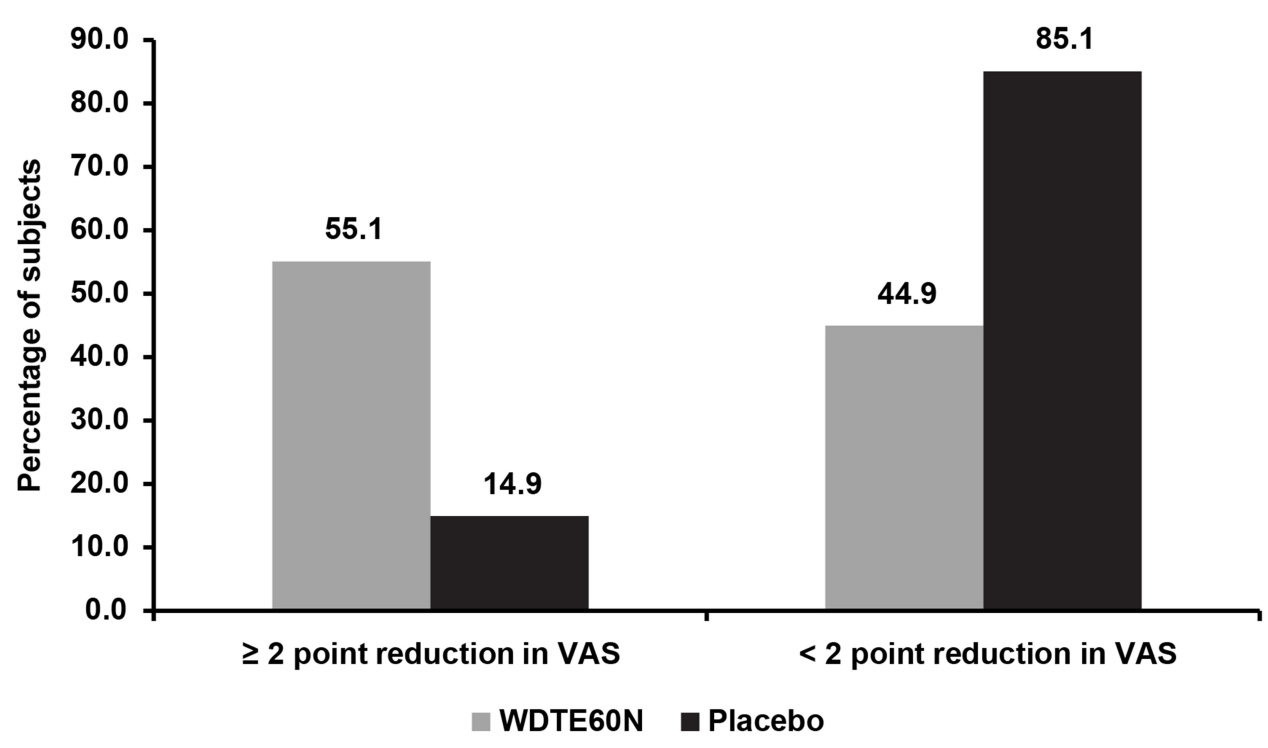

Figure 2 Proportion of subjects with reduction in VAS at day 90 .

C-reactive protein (CRP), IL-6, tumor necrosis factoralpha (TNF- $\alpha$ ), and matrix metalloproteinase-3 (MMP-3).

Intensity of knee pain was assessed using a $10-\mathrm{cm}$ VAS. $^{35}$ VAS has been a reliable and validated tool for the measurement of OA knee pain. It was measured with asking the question "how much pain did you have after 80 meter Fast-Paced walk test". The beginning of the line illustrates "no pain" and the end of the line indicates the "worst imaginable pain". Improvement in the joint mobility performance was measured by the 80 -m fast-paced walk test: using a stopwatch, time taken in seconds to complete the walk was recorded. The 9-step SCT was conducted to evaluate knee function, lower body strength, and balance. Each subject was instructed to ascend and descend a 9-step staircase with a 20$\mathrm{cm}$ step height. Time (in seconds) taken to ascend and descend flight of stairs was recorded. Subjects were allowed to use handrail or walking aid and to stop or rest if needed, but the time was recorded continuously. Both the fast-paced walk test and Stair-climb tests are recommended tests by the Osteoarthritis Research Society International (OARSI) for the measurement of performance in knee OA ${ }^{36,37}$ Flexion in the primarily affected knee or primary knee flexion was measured using a goniometer (pal Surgical Works, New Delhi, India) and expressed in degrees $\left({ }^{\circ}\right) .^{38}$

CRP and IL-6 levels are reliable markers of inflammation. TNF- $\alpha$ is reported to contribute critically to joint destruction in OA. MMP-3 has a key role in the proteolysis of proteoglycans that releases glycosaminoglycancontaining fragments (GAGs) that is an early feature of cartilage breakdown in OA. ${ }^{39-42} \mathrm{CRP}$ was measured by tubidometrically using Biosystem BA400 analyzer and test kits (Barcelona, Spain). Other markers were measured by an ELISA on CARETIUM KC100 Micro Plate Reader using Biosystem commercial kits.

\section{Safety Assessments}

Analyses of a set of hematological and biochemical parameters, as well as urine analysis, were performed at screening, and at the end of the study to ensure the safety of the study subjects - hematology using ABX Pentra XL80, Biochemistry using Biosystem BA400 and Urine Analysis using routine analysis manual. Vital signs and physical examinations were performed at all visits. Any AE occurring during the study was reported.

\section{Statistical Analysis}

Power analysis estimated that at least 106 subjects would be required to be enrolled with following assumptions: a power of $80 \%$, mean difference in pain score: 1.444 , standard deviation: 2.5 , and a dropout rate: $10 \%$.

Analyses on the intention-to-treat (ITT) population presented the efficacy and safety of the study product. Summary statistics for all parameters was performed, and the results were presented as mean \pm standard deviation for continuous variables and as count and percentages for categorical variables. Data were not normally distributed, as determined by the Kolmogorov-Smirnov test. Hence, Wilcoxon signed-rank test was used to compare data within groups and the Mann-Whitney $U$-test was used to 
Table 3 Biochemical and Inflammatory Markers

\begin{tabular}{|c|c|c|c|c|c|}
\hline & \multicolumn{2}{|c|}{ WDTE60N $(n=49)$} & \multicolumn{2}{|c|}{ Placebo $(n=47)$} & \multirow[t]{2}{*}{$p$-value } \\
\hline & Mean \pm SD & Median (IQR) & Mean \pm SD & Median (IQR) & \\
\hline \multicolumn{6}{|c|}{ C-reactive protein $(\mathrm{mg} / \mathrm{dL})$} \\
\hline \multirow{4}{*}{$\begin{array}{l}\text { Baseline } \\
\text { Day } 90 \\
\text { Change from B to D90 } \\
\text { p-value** }\end{array}$} & $5.3 \pm 14.0$ & $\mathrm{I} .7(0.9,4.8)$ & $3.0 \pm 3.6$ & $1.6(0.8,3.4)$ & \\
\hline & $2.6 \pm 2.6$ & $1.6(0.9,3.4)$ & $9.5 \pm 26.8$ & $1.9(1,4)$ & \\
\hline & $-2.7 \pm 14.5$ & $-0.2(-1.5,1.1)$ & $6.5 \pm 26.7$ & $0.0(-0.8,1.4)$ & 0.2868 \\
\hline & \multicolumn{2}{|l|}{0.2327} & \multicolumn{2}{|l|}{0.2676} & \\
\hline \multicolumn{6}{|l|}{ Interleukin $6(\mathrm{pg} / \mathrm{mL})$} \\
\hline \multirow{4}{*}{$\begin{array}{l}\text { Baseline } \\
\text { Day } 90 \\
\text { Change from B to D90 } \\
\text { p-value** }\end{array}$} & $21.1 \pm 31.3$ & $8.1(0.07,29.7)$ & $20.5 \pm 36.3$ & $3.3(0.07,18.4)$ & \\
\hline & $30.5 \pm 52.6$ & 4.I $(0.7,26.7)$ & $26.8 \pm 40.2$ & $8.3(I, 31.7)$ & \\
\hline & $9.4 \pm 62.7$ & $0.5(-19,12.8)$ & $6.3 \pm 36.6$ & $3.1(-0.79,22.6)$ & 0.1699 \\
\hline & \multicolumn{2}{|l|}{$0.464 I$} & \multicolumn{2}{|l|}{0.0188} & \\
\hline \multicolumn{6}{|c|}{ Tumor necrosis factor- $\alpha(\mathrm{pg} / \mathrm{mL})$} \\
\hline \multirow{4}{*}{$\begin{array}{l}\text { Baseline } \\
\text { Day } 90 \\
\text { Change from B to D90 } \\
p \text {-value** }\end{array}$} & $30.4 \pm 52.5$ & $2.6(0.2,48.8)$ & $31.6 \pm 60.6$ & $0.7(0.1,34.4)$ & \\
\hline & $14.9 \pm 45.1$ & $0.7(0.7,0.7)$ & $22.4 \pm 32.6$ & $0.7(0.7,30.7)$ & \\
\hline & $-15.5 \pm 67.1$ & $-0.2(-24.9,0.6)$ & $-9.3 \pm 67.3$ & $0.5(-20.6,12.4)$ & 0.0927 \\
\hline & \multicolumn{2}{|l|}{0.0054} & \multicolumn{2}{|l|}{0.4522} & \\
\hline \multicolumn{6}{|c|}{ Matrix metalloproteinase-3 $(\mathrm{ng} / \mathrm{mL})$} \\
\hline \multirow{4}{*}{$\begin{array}{l}\text { Baseline } \\
\text { Day } 90 \\
\text { Change from B to D } 90 \\
\text { p-value** }\end{array}$} & $20.9 \pm 15.4$ & $19.7(6.6,35.0)$ & $18.9 \pm 16.6$ & $12.5(5.5,34.2)$ & \\
\hline & $14.1 \pm 9.4$ & $12.5(6.6,19.6)$ & $15.9 \pm 9.7$ & $13(7.7,23.5)$ & \\
\hline & $-6.8 \pm 14.9$ & $-7(-15.7,2.5)$ & $-3.0 \pm 14$ & $-0.4(-9.4,5.7)$ & 0.1387 \\
\hline & \multicolumn{2}{|l|}{0.0017} & \multicolumn{2}{|l|}{0.1335} & \\
\hline
\end{tabular}

Notes: ${ }^{*} p$-value from the Mann-Whitney $U$-test comparing the difference between placebo versus WDTE60N; ${ }^{*} p$-value from Wilcoxon signed-rank test comparing levels at baseline to day 90 .

Abbreviations: $B$ to $D$, baseline to day; IQR, interquartile range.

compare data between test and placebo groups. A $p<0.05$ was considered to indicate statistical significance.

\section{Results}

Of the 121 subjects screened for inclusion in the study, 106 subjects were randomized such that each treatment arm had 53 subjects. Furthermore, 10 subjects were lost to follow-up (6 in placebo and 4 in WDTE60N group); thus, 96 subjects completed the study (Figure 1). The demographic characteristics at baseline were similar between both groups (Table 1).

Table 2 lists the changes in efficacy parameters. A statistically significant reduction in the intensity of pain was noted on VAS: from $5.4 \pm 0.9$ at baseline to $3.8 \pm 0.8$ at day 90 in the WDTE60N group. The mean reduction in pain intensity was greater in the WDTE60N group than in the placebo group $(1.5 \pm 0.7$ points vs $-0.6 \pm 0.8, p<$ $0.0001)$. Fifty-five percent of subjects reported $\geq 2$ point reduction in the pain VAS score with WDTE60N group compared to $14 \%$ subjects in the placebo group (Figure 2). Improvement of 2 points on VAS scale is considered the minimal clinically important difference. ${ }^{43-45}$

Furthermore, supplementation with WDTE60N led to improved performance in the 80 -m fast-paced walk test: from $70.3 \pm 23.9 \mathrm{~s}$ at baseline to $59.3 \pm 9.5 \mathrm{~s}$ at day 90 . The mean difference from baseline was $-11.0 \pm 26.1 \mathrm{~s}$ and $-6.8 \pm 23.3$ s for WDTE60N and placebo $(p=0.0011)$ groups, respectively, indicating that WDTE60N supplementation significantly improved mobility and performance in the $80-\mathrm{m}$ walk test.

Reduction in time taken by subjects to ascend and descend stairs in the 9-step SCT is considered as an efficacy measure. At the end of the study, time taken by the subjects in the SCT was reduced by $12.3 \pm 24.2 \mathrm{~s}$ in the WDTE60N group compared to $7.8 \pm 24.2 \mathrm{~s}$ in the placebo ( $p<0.0001)$, indicating improvement in the knee function, lower body strength, and balance.

Measurement of the knee flexion assesses the angular movement of the affected knee. Change from baseline 
knee flexion in WDTE60N and placebo groups were -0.2 $\pm 0.6^{\circ}$ and $-0.1 \pm 0.8^{\circ}$, respectively. The change was not statistically significant $(p=0.3657)$.

Overall, the significant improvement in efficacy variables was observed as early as day 45 (Table 2).

Analysis of four inflammatory biomarkers for change in levels from baseline to end of the study revealed no significant difference between the WDTE60N and placebo groups (Table 3). However, a clear trend of improvement was observed for all the four biomarkers in the WDTE60N group. In brief, there was no significant difference in the median (IQR) CRP levels at baseline and at day 90 day in the WDTE60N group $(1.7[0.9,4.8]$ vs $1.6[0.9,3.4], p=$ $0.2327)$ and placebo group $(1.6[0.8,3.4]$ vs $1.9[1.0,4.0]$, $p=0.2676$ ). The increase in the median IL-6 level was not significant in the WDTE60N $(8.1[0.07,29.7]$ vs $4.1[0.7$, 26.7], $p=0.4641$ ) group but it significantly increased in the placebo group (3.3 [0.07, 18.4] vs $8.3[1,31.7], p=0.0188)$. The median TNF- $\alpha$ level reduced significantly in WDTE60N group $(2.6[0.2,48.8]$ vs 0.7 [0.7, 0.7], $p=0.0054)$ whereas there was no statistical significant change in the placebo group $(0.7[0.1,34.4]$ vs $0.7[0.7,30.7], p=0.4522)$. Similarly, median MMP-3 level significantly decreased in the WDTE60N group $(19.7[6.6,35.0]$ vs $12.5[6.6,19.6]$, $p=0.0017)$ whereas there was no change in the placebo group $(12.5[5.5,34.2]$ vs 13 [7.7, 23.5], $p=0.1335)$.

Levels of various safety parameters (aspartate aminotransferase [AST], alanine aminotransferase [ALT], serum lipid profile, fasting glucose, serum creatinine, complete blood count [CBC], erythrocyte sedimentation rate [ESR]) were within the normal/optimal range at baseline, and both treatments did not show any changes in these parameters at the end of the treatment. No subjects used rescue medications throughout the study period. Three AEs occurred during the study and all were unrelated to the study treatments. No serious AE was observed during the study (Table 4).

\section{Discussion}

The present study showed that 3-month supplementation with WDTE60N improves joint comfort and alleviates

Table 4 Summary of Adverse Events

\begin{tabular}{|l|l|l|}
\hline Adverse Events & WDTE60N (n = 53) & Placebo (n = 53) \\
\hline Headache & $\mathrm{I}(1.89 \%)$ & 0 \\
Nausea & $\mathrm{I}(1.89 \%)$ & 0 \\
Diarrhea & 0 & $\mathrm{I}(1.89 \%)$ \\
\hline
\end{tabular}

chronic knee pain in healthy subjects, as indicated by reduction in the VAS score following physical exertion induced by the $80-\mathrm{m}$ fast-paced walk test. Joint performance and mobility also improved as shown by the 9 step SCT and 80 -m fastpaced walk test. Given the higher exposure of curcumin from the low-dose WDTE60N and the proven anti-inflammatory action of curcumin, WDTE60N could be an effective supplementation for improving joint function in the healthy population who may be at risk of developing OA.

Chronic knee pain can adversely affect daily activities like climbing of the stairs even in non-arthritic adults, and this could be a psycho-physiological limiting factor that may lead to avoidance of routine daily activities. ${ }^{46,47}$ It is reported that chronic knee pain can predict the structural progression to $\mathrm{OA}^{48}$ In the present study, WDTE60N significantly reduced pain on walking as measured on VAS from baseline at the end of 3 months supplementation period as compared to placebo. Minimal clinically relevant reduction in VAS scores, which is defined to be $\geq 2$ points, was attained in $>50 \%$ of the subjects in the WDTE60N group compared to $14 \%$ in the placebo group. Reduction in the VAS scores correlated with the 80-m walk test and the 9-step SCT. Range of motion as assessed by knee flexion test was within normal range and did not change further with both treatments. These observations suggest that WDTE60N can potentially improve joint comfort by alleviating knee pain that occurs during and after regular physical activity and, thus, also contribute to improvement in the overall performance of the affected joint.

The global burden of disease study 2017 showed a large burden of musculoskeletal disorders, with approximately 1.3 billion cases globally. ${ }^{49}$ Of these, the knee OA is among the most disabling musculoskeletal conditions. Inflammation is regarded as an important feature of early $\mathrm{OA}$, and the altered inflammatory state induced by mechanical stress is considered an underlying cause. $^{50}$ Patients with early OA with knee pain exhibit significantly higher levels of certain inflammatory markers such as TNF- $\alpha$, IL-1 $\beta$, IL-6, and CRP. ${ }^{6}$ No significant changes in inflammatory marker levels for both the WDTE60N and placebo groups were observed in our study. Since the study subjects were healthy and without any evident arthritis, the inflammatory markers were within normal range at the beginning of the study. Another study by Nieman et al has also reported similar results in healthy adults. ${ }^{51}$ However, the extent of improvement in the inflammatory biomarker levels was 
higher in the WDTE60N group, and this is an important finding as it may implicate the prevention of further worsening of knee joint pain.

Although curcumin as a dietary supplement has proven benefits in patients with OA with equal efficacy and much lower incidence of side effects than NSAIDs, its use is limited by poor bioavailability, ${ }^{22}$ which mandates the administration of multiple daily doses, resulting in low compliance on long-term administration. Low bioavailability of turmeric has led to extensive research, which has flooded the market with turmeric formulations manufactured using various technologies. ${ }^{52}$ Most marketed bioavailable turmeric formulations have shown significantly improved bioavailability in preclinical and clinical PK studies as compared to standard turmeric extract $95 \%,{ }^{53-55}$ however in their clinical evaluation; these formulations are not used in the dose found to be more bioavailable in PK studies. ${ }^{52-54,56}$ On the other hand, in this study we used the low-dose of WDTE60N (250 mg with $150 \mathrm{mg}$ curcuminoids), which has exhibited the higher relative bioavailability of $150 \mathrm{mg}$ of curcuminoids as compared to the higher dose of standard $95 \%$ turmeric extract (with $1500 \mathrm{mg}$ curcuminoids) in the previous PK studies. Therefore, our study is a first-of-its-kind to evaluate the optimum dosage of our formulation through PK analysis and to show the efficacy of the same dose in the clinical study. This low-dose turmeric formulation alleviated pain and improved knee joint performance and mobility when administered over 3 months. Using low-dose turmeric formulation in a single daily administration may avoid safety concerns and compliance issues; it serves as an alternative to the contemporary higher-dose turmeric extract formulations and can be used for longer duration for joint protection in individuals with chronic knee pain.

Despite favorable results, the study had two limitations. First, this study was conducted on healthy adults. Second, there was no significant difference in the biomarkers between the groups. However, the improvement observed in pain scores and physical function was noteworthy. Studies with a larger sample size in patients with knee OA would be of future research interest.

\section{Conclusion}

In summary, 3-months supplementation with WDTE60N $250 \mathrm{mg}$ once daily improved joint comfort by alleviating chronic knee pain and also improved joint performance and mobility in healthy subjects with chronic knee pain. It also showed improvement (although non-significant) in inflammatory biomarker levels. Furthermore, WDTE60N was well tolerated and found to be safe for human consumption.

\section{Abbreviations}

AEs, adverse events; ALT, alanine aminotransferase; AST, aspartate aminotransferase; BDMC, bisdemethoxycurcumin; BMI, body mass index; CBC, complete blood count; CRP, C-reactive protein; DMC, demethoxycurcumin; ESR, erythrocyte sedimentation rate; GAGs, glycosaminoglycan-containing fragments; GCP, Good Clinical Practice; IL, interleukin; ITT, intention-to-treat; MMP-3, matrix metalloproteinase-3; NSAIDs, Non-steroidal antiinflammatory drugs; OA, Osteoarthritis; OARSI, Osteoarthritis Research Society International; PK, pharmacokinetics; SCT, stair climb test; TNF- $\alpha$, tumor necrosis factor-alpha VAS, visual analogue scale.

\section{Institutional Review Board Statement}

The study was conducted according to the guidelines of the Declaration of Helsinki, and approved by the Ethics Committee of Sri Venkateshwara Hospital Ethics Committee (protocol code PRO-INV-012-019 and date of approval: 18 February 2020) and Shetty's Hospital Ethics Committee (protocol code PRO-INV-012-019 and date of approval: 18 February 2020).

\section{Data Sharing Statement}

The data presented in this study are available on request from the corresponding author.

\section{Informed Consent Statement}

All subjects provided written informed consent prior initiating clinical study-related activities.

\section{Acknowledgments}

The authors would like to acknowledge CBCC Global Research for their writing and editorial support in the development of this manuscript which was funded by Inventia Healthcare Ltd.

\section{Author Contributions}

All authors made a significant contribution to the work reported, whether that is in the conception, study design, execution, acquisition of data, analysis and interpretation, or in all these areas; took part in drafting, revising or critically reviewing the article; gave final approval of the 
version to be published; have agreed on the journal to which the article has been submitted; and agree to be accountable for all aspects of the work.

\section{Funding}

This research was funded by Inventia Healthcare Ltd., India, and Laila Nutraceuticals India.

\section{Disclosure}

Shefali Thanawala and Rajat Shah are employees of Inventia Healthcare Ltd. KrishnaRaju Venkata Alluri and Venkateswarlu Somepalli are employees of Laila Nutraceuticals. Prabakaran Desomayanandam and Arun Bhuvanendran are employees of In Vitro Research Solutions (iVRS) Pvt Ltd. The authors do not have any other conflicts of interest to declare.

\section{References}

1. Rao PS, Ramanjaneyulu YS, Prisk VR, Schurgers LJ. A combination of tamarindus indica seeds and curcuma longa rhizome extracts improves knee joint function and alleviates pain in non-arthritic adults following physical activity. Int $J$ Med Sci. 2019;16 (6):845-853. doi:10.7150/ijms.32505

2. Frese T, Peyton L, Mahlmeister J, Sandholzer H. Knee pain as the reason for encounter in general practice. ISRN Family Med. 2013;2013:930825. doi:10.5402/2013/930825

3. Jinks C, Jordan K, Ong B, Croft P. A brief screening tool for knee pain in primary care (KNEST). 2. Results from a survey in the general population aged 50 and over. Rheumatology (Oxford). 2004;43(1):55-61. doi:10.1093/rheumatology/keg438

4. Neogi T. The epidemiology and impact of pain in osteoarthritis Osteoarthritis Cartilage. 2013;21(9):1145-1153. doi:10.1016/j. joca.2013.03.018

5. Antony B, Jones G, Jin X, Ding C. Do early life factors affect the development of knee osteoarthritis in later life: a narrative review. Arthritis Res Ther. 2016;18(1):202. doi:10.1186/s13075-016-1104-0

6. Mabey T, Honsawek S. Cytokines as biochemical markers for knee osteoarthritis. World J Orthop. 2015;6(1):95-105. doi:10.5312/wjo. v6.i1.95

7. Goldring MB, Otero M. Inflammation in osteoarthritis. Curr Opin Rheumatol. 2011;23(5):471-478. doi:10.1097/BOR.0b013e328349c2b1

8. Ghasemian M, Owlia S, Owlia MB. Review of anti-inflammatory herbal medicines. Adv Pharmacol Sci. 2016;2016:9130979. doi:10.1155/2016/9130979

9. Kraus V, BirminghamJ, Stabler T, et al. Effects of intraarticular IL1-Ra for acute anterior cruciate ligament knee injury: a randomized controlled pilot trial (NCT00332254). Osteoarthritis Cartilage. 2012;20(4):271-278. doi:10.1016/j.joca.2011.12.009

10. Jones BQ, Covey CJ, Sineath MH Jr. Nonsurgical management of knee pain in adults. Am Fam Physician. 2015;92(10):875-883.

11. Risser A, Donovan D, Heintzman J, Page T. NSAID prescribing precautions. Am Fam Physician. 2009;80(12):1371-1378.

12. Fine M. Quantifying the impact of NSAID-associated adverse events. Am J Manag Care. 2013;19(14 Suppl):s267-s272.

13. Wongrakpanich S, Wongrakpanich A, Melhado K, Rangaswami J. A comprehensive review of non-steroidal anti-inflammatory drug use in the elderly. Aging Dis. 2018;9(1):143-150. doi:10.14336/ AD.2017.0306
14. Taylor SD, Everett SV, Taylor TN, Watson DJ, Taylor-Stokes G. A measure of treatment response: patient and physician satisfaction with traditional NSAIDs for osteoarthritis control. Open Access Rheumatol. 2013;5:69-76. doi:10.2147/OARRR.S41940

15. Laires PA, Laíns J, Miranda LC, et al. Inadequate pain relief among patients with primary knee osteoarthritis. Rev Bras Reumatol Engl Ed. 2017;57(3):229-237. doi:10.1016/j.rbre.2016.11.005

16. Shirley PY, Hunter DJ. Managing osteoarthritis. Aust Prescr. 2015;38 (4):115-119. doi:10.18773/austprescr.2015.039

17. Henrotin Y, Lambert C, Couchourel D, Ripoll C, Chiotelli E. Nutraceuticals: do they represent a new era in the management of osteoarthritis?-a narrative review from the lessons taken with five products. Osteoarthritis Cartilage. 2011;19(1):1-21. doi:10.1016/j. joca.2010.10.017

18. Di Paola R, Fusco R, Impellizzeri D, et al. Adelmidrol, in combination with hyaluronic acid, displays increased anti-inflammatory and analgesic effects against monosodium iodoacetate-induced osteoarthritis in rats. Arthritis Res Ther. 2016;18(1):291. doi:10.1186/s13075-016-1189-5

19. Fusco R, Siracusa R, Peritore AF, et al. The role of cashew (Anacardium occidentale L.) nuts on an experimental model of painful degenerative joint disease. Antioxidants (Basel). 2020;9(6):511. doi:10.3390/antiox9060511

20. Castrogiovanni P, Trovato FM, Loreto C, et al. Nutraceutical supplements in the management and prevention of osteoarthritis. Int $J \mathrm{Mol}$ Sci. 2016;17(12):2042. doi:10.3390/ijms17122042

21. Liu X, Machado GC, Eyles JP, Ravi V, Hunter DJ. Dietary supplements for treating osteoarthritis: a systematic review and meta-analysis. Br J Sports Med. 2018;52(3):167-175. doi:10.1136/bjsports-2016-097333

22. Bannuru RR, Osani MC, Al-Eid F, Wang C. Efficacy of curcumin and boswellia for knee osteoarthritis: systematic review and meta-analysis. Semin Arthritis Rheum. 2018;48(3):416-429. doi:10.1016/j.semarthrit.2018.03.001

23. Onakpoya IJ, Spencer EA, Perera R, Heneghan CJ. Effectiveness of curcuminoids in the treatment of knee osteoarthritis: a systematic review and meta-analysis of randomized clinical trials. Int $J$ Rheum Dis. 2017;20(4):420-433. doi:10.1111/1756-185X.13069

24. Daily JW, Yang M, Park S. Efficacy of turmeric extracts and curcumin for alleviating the symptoms of joint arthritis: a systematic review and meta-analysis of randomized clinical trials. $J$ Med Food. 2016;19(8):717-729. doi:10.1089/jmf.2016.3705

25. Aghili Khorasani MH. Makhzan-Al-Adviah (Rewritten by Shams Ardakani MR, Rahimi R, Farjadmand F). 1st ed. Tehran: Tehran University of Medical Sciences; 1771.

26. Ahmad RS, Hussain MB, Sultan MT, et al. Biochemistry, safety, pharmacological activities, and clinical applications of turmeric: a mechanistic Review. Evid Based Complement Alternat Med. 2020;2020:7656919. doi:10.1155/2020/7656919

27. Payton F, Sandusky P, Alworth WL. NMR study of the solution structure of curcumin. $J$ Nat Prod. 2007;70(2):143-146. doi:10.1021/np060263s

28. Li X, Feng K, Li J, et al. Curcumin inhibits apoptosis of chondrocytes through activation ERK1/2 signaling pathways induced autophagy. Nutrients. 2017;9(4):414. doi:10.3390/nu9040414

29. Liacini A, Sylvester J, Li WQ, Zafarullah M. Inhibition of interleukin1-stimulated MAP kinases, activating protein-1 (AP-1) and nuclear factor kappa B (NF-kB) transcription factors down-regulates matrix metalloproteinase gene expression in articular chondrocytes. Matrix Biol. 2002;21 (3):251-262. doi:10.1016/S0945-053X(02)00007-0

30. Mathy-Hartert M, Jacquemond-Collet I, Priem F, et al. Curcumin inhibits pro-inflammatory mediators and metalloproteinase- 3 production by chondrocytes. Inflamm Res. 2009;58(12):899-908. doi:10.1007/s00011-009-0063-1

31. Mun SH, Kim HS, Kim JW, et al. Oral administration of curcumin suppresses production of matrix metalloproteinase (MMP)-1 and MMP-3 to ameliorate collagen-induced arthritis: inhibition of the $\mathrm{PKC} / \mathrm{JNK} / \mathrm{c}-\mathrm{Jun}$ pathway. J Pharmacol Sci. 2009;111(1):13-21. doi:10.1254/jphs.09134FP 
32. Liu W, Zhai Y, Heng X, et al. Oral bioavailability of curcumin: problems and advancements. J Drug Target. 2016;24(8):694-702. doi:10.3109/1061186X.2016.1157883

33. Fadus MC, Lau C, Bikhchandani J, Lynch HT. Curcumin: an age-old anti-inflammatory and anti-neoplastic agent. J Tradit Complement Med. 2017;7(3):339-346. doi:10.1016/j.jtcme.2016.08.002

34. Thanawala S. New technologies to increase the bioavailability of ayurvedic and nutraceutical ingredients. Paper presented at II international scientific conference RUDN University. Moscow, Russia; November 2019.

35. Alghadir AH, Anwer S, Iqbal A, Iqbal ZA. Test-retest reliability, validity, and minimum detectable change of visual analog, numerical rating, and verbal rating scales for measurement of osteoarthritic knee pain. J Pain Res. 2018;11:851-856. doi:10.2147/JPR.S158847

36. Dobson F, Hinman RS, Roos EM, et al. OARSI recommended performance-based tests to assess physical function in people diagnosed with hip or knee osteoarthritis. Osteoarthritis Cartilage. 2013;21(8):1042-1052. doi:10.1016/j.joca.2013.05.002

37. Dobson F, Hinman RS, Hall M, et al. Measurement properties of performance-based measures to assess physical function in hip and knee osteoarthritis: a systematic review. Osteoarthritis Cartilage. 2012;20(12):1548-1562. doi:10.1016/j.joca.2012.08.015

38. Steultjens M, Dekker J, Van Baar M, Oostendorp R, Bijlsma J. Range of joint motion and disability in patients with osteoarthritis of the knee or hip. Rheumatology (Oxford). 2000;39(9):955-961. doi:10.1093/rheumatology/39.9.955

39. Sproston NR, Ashworth JJ. Role of C-reactive protein at sites of inflammation and infection. Front Immunol. 2018;9:754. doi:10.3389/fimmu.2018.00754

40. Rincon M. Interleukin-6: from an inflammatory marker to a target for inflammatory diseases. Trends Immunol. 2012;33(11):571-577. doi:10.1016/j.it.2012.07.003

41. Pengas I, Eldridge S, Assiotis A, et al. MMP-3 in the peripheral serum as a biomarker of knee osteoarthritis, 40 years after open total knee meniscectomy. J Exp Orthop. 2018;5(1):21. doi:10.1186/s40634-018-0132-x

42. Stannus O, Jones G, Cicuttini F, et al. Circulating levels of IL-6 and $\mathrm{TNF}-\alpha$ are associated with knee radiographic osteoarthritis and knee cartilage loss in older adults. Osteoarthritis Cartilage. 2010;18 (11):1441-1447. doi:10.1016/j.joca.2010.08.016

43. Concoff A, Rosen J, Fu F, et al. A comparison of treatment effects for nonsurgical therapies and the minimum clinically important difference in knee osteoarthritis: a systematic Review. JBJS Rev. 2019;7 (8):e5. doi:10.2106/JBJS.RVW.18.00150

44. Hawker GA, Mian S, Kendzerska T, French M. Measures of adult pain: Visual Analog Scale for Pain (VAS Pain), Numeric Rating Scale for Pain (NRS Pain), McGill Pain Questionnaire (MPQ), Short-Form McGill Pain Questionnaire (SF-MPQ), Chronic Pain Grade Scale (CPGS), Short Form-36 Bodily Pain Scale (SF-36 BPS), and measure of Intermittent and Constant Osteoarthritis Pain (ICOAP). Arthritis Care Res (Hoboken). 2011;63(Suppl 11):S240 S252. doi:10.1002/acr.20543
45. Katz NP, Paillard FC, Ekman E. Determining the clinical importance of treatment benefits for interventions for painful orthopedic conditions. J Orthop Surg Res. 2015;10(1):24. doi:10.1186/s13018-014-0144-x

46. Leeuw M, Goossens ME, Linton SJ, et al. The fear-avoidance model of musculoskeletal pain: current state of scientific evidence. J Behav Med. 2007;30(1):77-94. doi:10.1007/s10865-006-9085-0

47. Holla JF, van der Leeden M, Heymans MW, et al. Three trajectories of activity limitations in early symptomatic knee osteoarthritis: a 5-year follow-up study. Ann Rheum Dis. 2014;73(7):1369-1375. doi:10.1136/annrheumdis-2012-202984

48. Wang Y, Teichtahl AJ, Abram F, et al. Knee pain as a predictor of structural progression over 4 years: data from the osteoarthritis initiative, a prospective cohort study. Arthritis Res Ther. 2018;20 (1):250. doi:10.1186/s13075-018-1751-4

49. Safiri S, Kolahi AA, Cross M, et al. Prevalence, deaths and disability adjusted life years (DALYs) due to musculoskeletal disorders for 195 countries and territories 1990-2017. Arthritis Rheumatol. 2020;72 (11):1916-1927. doi:10.1002/art.41571

50. Kraus VB, Blanco FJ, Englund M, Karsdal MA, Lohmander LS. Call for standardized definitions of osteoarthritis and risk stratification for clinical trials and clinical use. Osteoarthritis Cartilage. 2015;23 (8):1233-1241. doi:10.1016/j.joca.2015.03.036

51. Nieman DC, Shanely RA, Luo B, et al. A commercialized dietary supplement alleviates joint pain in community adults: a double-blind, placebo-controlled community trial. Nutr J. 2013;12(1):1-9. doi:10.1186/1475-2891-12-154

52. Liu Z, Smart JD, Pannala AS. Recent developments in formulation design for improving oral bioavailability of curcumin: a review. J Drug Deliv Sci Technol. 2020;60:102082. doi:10.1016/j. jddst.2020.102082

53. Cuomo J, Appendino G, Dern AS, et al. Comparative absorption of a standardized curcuminoid mixture and its lecithin formulation. J Nat Prod. 2011;74(4):664-669. doi:10.1021/np1007262

54. Kanai M, Imaizumi A, Otsuka Y, et al. Dose-escalation and pharmacokinetic study of nanoparticle curcumin, a potential anticancer agent with improved bioavailability, in healthy human volunteers. Cancer Chemother Pharmacol. 2012;69(1):65-70. doi:10.1007/s00280-011-1673-1

55. Jäger RLR, Calvanese AV, Joy JM, Purpura M, Wilson JM. Comparative absorption of curcumin formulations. Nutr J. 2014;13 (1):11. doi:10.1186/1475-2891-13-11

56. Nakagawa Y, Mukai S, Yamada S, et al. Short-term effects of highly-bioavailable curcumin for treating knee osteoarthritis: a randomized, double-blind, placebo-controlled prospective study. $J$ Orthop Sci. 2014;19(6):933-939. doi:10.1007/s00776-014-0633-0
Clinical Pharmacology: Advances and Applications is an international, peer-reviewed, open access journal publishing original research, reports, reviews and commentaries on all areas of drug experience in humans. The manuscript management system is completely online and includes a very quick and fair peer-review system, which is all easy to use. Visit http://www.dovepress.com/testimonials.php to read real quotes from published authors. 